\title{
Mechanistic investigation on the oxygen transfer with the manganese-salen complex
}

\author{
Thomas Bogaerts ${ }^{[a, b]}$, Sebastian Wouter $s^{[a, c]}$, Pascal Van Der Voort ${ }^{*[b]}$, Veronique Van Speybroeck ${ }^{*[a]}$
}

Abstract: The most well-known application of salen complexes is the use of a chiral ligand loaded with manganese to form the Jacobsen complex. This organometallic catalyst is used in the epoxidation of unfunctionalized olefins and can achieve very high selectivities. Although this application was proposed many years ago, the mechanism of oxygen transfer remains a question until now. In this paper, the epoxidation mechanism is investigated by an ab initio kinetic modeling study. First of all a proper DFT functional is selected which yields the correct ordering of the various spin states. Our results show that the epoxidation proceeds via a radical intermediate. Starting from the radical intermediate, these results can explain the experiments with radical probes. The subtle influences in the transition state using the full Jacobsen catalyst explain the experimentally observed product distribution.

\section{Introduction}

Since their discovery by Jacobsen and Katsuki, ${ }^{[1]}$ chiral manganese-salen complexes (Figure 1) have been an important tool for many organic chemists ${ }^{[2]}$. These manganese complexes allow the epoxidation of unfunctionalized olefins with an unprecedented conversion and selectivity. Especially conjugated olefins can be quantitatively converted with this system ${ }^{[3]}$. Other substrates such as thio-ethers and secondary alcohols have also been successfully oxidized with a high selectivity using salen-type complexes featuring different metals or modifications on the backbone ${ }^{[4]}$. The compatibility of this catalyst with different oxidizing agents, both in a watery as well as in a waterfree medium is another strong feature. The use of sodiumhypochloride ${ }^{[5]}$ as a cheap oxidant and the possibility of producing chiral salen catalysts on a ton $\operatorname{scale}^{[3 a, 6]}$ have made this catalytic system very interesting for the chemical industry.

Salen complexes in general consist of a diamine backbone connected with two salicylaldehyde moieties (Figure 1) and are

[a] Prof Dr. V. Van Speybroaeck, T. Bogaerts,

Center for Molecular Modelling (CMM)

Ghent university

Technologiepark 903,9052 Zwijnaarde, Belgium

E-mail: Veronique.vanspeybroeck@ugent.be

[b] Prof Dr. P. Van Der Voort

Center for Ordered Materials, Organometallics and Catalysis

(COMOC), Department of Inorganic and Physical Chemistry

Ghent University

Krijgslaan 281-S3, 9000 Ghent, Belgium

[c] Dr S. Wouters

Department of Chemistry

Princeton University

Frick Laboratory 351, Princeton, NJ 08544, USA

Supporting information for this article is given via a link at the end of the document. loaded with various metals. Different functional groups on the salicylaldehyde moieties can influence the activity and selectivity of the resulting catalyst ${ }^{[7]}$. When the backbone consists of a chiral diamine such as ethylenediamine, cyclohexanediamine or diphenylethylene-diamine, the complex can be used to catalyze various enantioselective reactions. The most well-known variant of this catalyst consists of a cyclohaxanediamine backbone, two 3,5-tert-butylsalecylaldehyde 'wings' and is loaded with manganese. This is the so-called Jacobsen catalyst ${ }^{[8]}$.

(ii)
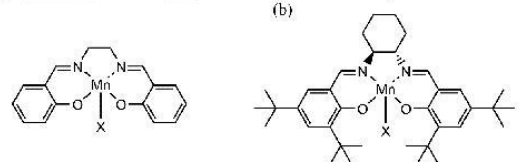

Figure 1. Motif of salen type complexes in general (a) and the arguably most well-known chiral variant: the Jacobsen complex.

The mechanism of olefin epoxidation with these complexes has been a topic for debate for many years. An important breakthrough was the isolation of the $\mathrm{Mn}^{\mathrm{V}}$-oxo intermediate with the use of mass spectroscopy ${ }^{[9]}$. Starting from this intermediate a two-step mechanism seemed the most plausible (Scheme 1(B)), this was supported by the observation that cis-olefins lead to both cis- and trans-epoxides ${ }^{[3 c]}$. This isomerization suggests there is some sort of intermediate where a free rotation around the $\mathrm{C}-\mathrm{C}$ bond is possible. In the two-step mechanism (Scheme $1(B))$ the first carbon-oxygen bond is formed leading to a radical intermediate, in this intermediate a rotation around the olefin C$C$ bond is possible which would explain the experimental observations. In a next step the intermediate collapses with the formation of the second $\mathrm{C}-\mathrm{O}$ bond.

However, this straightforward explanation was quickly questioned by experimental results from Wong et al. ${ }^{[10]}$ They proposed to investigate the mechanism further using a radical probe, this is a molecule where a three-membered ring is placed vicinal to the double bond that is epoxidized. The hypothesis of the authors was that any radical intermediate that appeared would be 'captured' by the opening of this unstable ring. The approach was further optimized by Linde et al. ${ }^{[11]}$ The probes used in their study were improved with strategically placed phenyl rings to improve the radical stability (Figure 2). To ensure an unbiased result, a molecule was chosen that had phenyl groups to stabilize both radicals (ring-opened and intermediate) equally.

The results of this latter study were surprising; different substituents on the one side of the double bond yielded different distributions of ring-opening and epoxide products (Scheme 2 and Table 1). Those observations lead to the conclusion that two different mechanisms for the epoxidation would be operating 
parallel to each other, the first featuring a radical intermediate, the second one via a different approach.

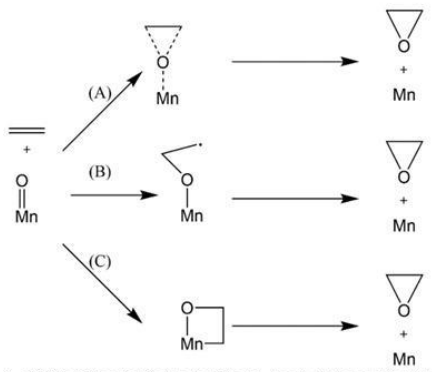

Scheme 1. Different mechanisms that have been proposed for the oxygen transfer from the manganese-salen complex; The concerted (A), radical (B) and manganaoxetane $(\mathrm{C})$ pathway.

The possible existence of a different mechanism has led to numerous ab initio studies to unravel the mechanism of the oxygen transfer. Initial results already showed that the spin state of the complex will play an important role in how exactly the transfer takes place ${ }^{[12]}$. By using a B3LYP functional and reducing the salen-type complex to a cationic Manganeseacacen system as a model Linde et al. ${ }^{[12 a]}$ found the existence of a concerted pathway (Scheme $1(A)$ ) that was more stable on the spin two plane, while for the spin 1 plane a two-step mechanism with a radical intermediate was found the most favored. Abashkin et al. ${ }^{[13]}$ found that the addition of a counter ion on the manganese center radically shifted the results. A two-step mechanism was found for both the spin 1 and 2 states while a concerted mechanism was proposed for the spin zero state. Next to the use of a more realistic model featuring a counter ion, the BP86 functional was used since it agreed well with the high level $\operatorname{CCSD}(T)$ method. Using this functional, the group of Jacobsen further analyzed this interesting reaction ${ }^{[14]}$ while considering various alternative intermediates next to the radical variant. Using this analysis Cavallo et al. ${ }^{[15]}$ proposed a full catalytic cycle for the epoxidation with sodiumhypochloride as an oxidant. Next to the radical intermediate other intermediates (e.g Scheme $1(C)$ ) were proposed in various modeling studies, ${ }^{[12 a, 16]}$ however, no full pathway via these intermediates was reported.

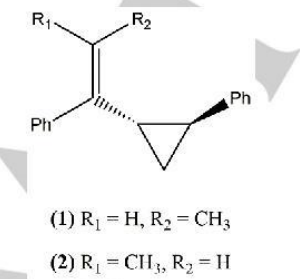

Figure 2 Two variants of the radical probe used by Linde et al ${ }^{[11 b]}$ to assess the existence of a radical intermediate.

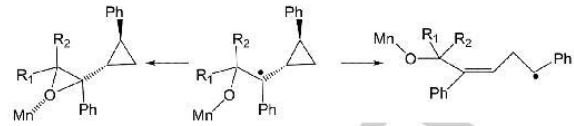

Scheme 2. Competitive ring-opening and epoxidation pathways with the radical probe experiments.

Despite these many interesting results the question of the influence of the selected density functional remained. Due to the near-degenerate spin states, the electronic description of this complex is a challenge. For that reason, CASSCF calculations are often proposed to model the active species in the epoxidation with the salen complex ${ }^{[17]}$. This method has shown that the $\mathrm{Mn}^{\mathrm{V}}$-oxo complex (Figure 3 ) has a high multireference character, thus static correlation should be adequately described In an earlier study we reported that the DMRG-SCF method ${ }^{[18]}$ allows to model this multireference system with high accuracy ${ }^{[19]}$ With that approach a very good estimation of the electronic structure and relative free energy of the different spin states for the $\mathrm{Mn}^{\mathrm{V}}$-oxo complex was achieved.

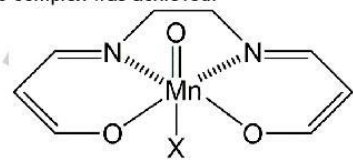

Figure 3. $\mathrm{Mn}^{\mathrm{V}}$-oxo structure on the Mn-acacen' complex.

On the other hand, we also have shown earlier that a proper description of the full catalyst (similar to Figure $1(b)$ ) is necessary to obtain a proper description of the selectivity ${ }^{[20]}$. It can be expected that the large model will be required here as well. This large model cannot be modeled with these high-leve post-HF methods. In this paper we aim to unravel the epoxidation mechanism by the means of an advanced theoretical approach. Therefore we have first investigated whether a good density functional can be found that allows to reproduce the relative free energies of the different spin states of the $\mathrm{Mn}^{\mathrm{V}}$-oxo acacen' complex obtained by the DMRG-SCF method. Secondly the oxygen transfer mechanism will be assessed on the small complex, using ethene as a test substrate. Finally the oxygen transfer with the radical probe as a substrate will be considered to find the mechanistic explanation for the distribution between ring-opening and epoxidation products. In this case the full model for the catalyst will be used.

Table 1. Experimentally observed distribution percentage of epoxide formation. [11 1b]

$\mathrm{NaOCl} / \mathrm{CH} 2 \mathrm{Cl} 2$ (a) lodosylbenzene/ benzene (b)

\begin{tabular}{llll}
\hline$R_{1}=H R_{2}=M e$ & (1) & $100 \%$ & $83 \%$ \\
$R_{1}=M e R_{2}=H$ & (2) & $56 \%$ & $54 \%$ \\
\hline
\end{tabular}




\section{Results and Discussion}

Computational description of the salen complex

To model the full catalyst we aim to use density functiona methods. Thus the selection of a proper functional has to be investigated first. Since the possible spin states during the reaction are energetically close to each other, an important feature of a good functional is its capability of finding the right energetic ordering of the various spin states. As a reference we will use the energies found by the DMRG-SCF approach with a $6-31 \mathrm{G}^{*}$ basis set for the $\mathrm{Mn}^{\mathrm{V}}$-oxo acacen' complex ${ }^{[18-19]}$. Using this method it was possible to obtain a multireference result with a very large active space. The spin 1 state was found to be the most stable, followed by the spin 0 and spin 2 state (DMRGSCF in Figure 4). Herein, single point energy calculations were done on the same geometry with the same basis set and using a broad scale of functionals to assess the capability of the functional to describe the right spin ordering. The energy differences relative to the spin 0 state were compared to the reference DMRGSCF calculations (Figure 4).

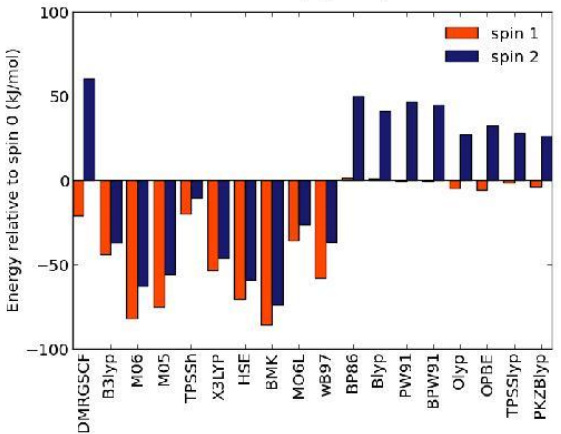

Figure 4. Energies of various spin states with a selected set of for the acacen complex.

From this comparison some trends could be deduced, there is a whole range of functionals that overestimate the high spin states. Especially functionals containing Hartree-fock exchange exhibit this behavior. A few functionals, including the BP86 functional seem to overestimate the stability of the spin 0 state, however the results are closer to the multireference values. The next set of functionals (OLYP, OPBE,...) reproduce the correct ordering but not the numerical values. The best results seem to come from the functionals containing Handy's ${ }^{[21]}$ modification of the Becke exchange functional (OPTX). From this benchmark we have selected the OPBE functional as the preferred method. These results are also supported by the success of this functional in the modelling of systems with a challenging electronic configuration where different spin states are energetically close to each other ${ }^{[22]}$

This level of theory will be used hereafter on three models to obtain mechanistic insight into the epoxidation reactions. First of all, the manganese-acacen' complex (Figure 3) will be investigated in combination with ethylene as olefin. This is a very small model system, which allows to quickly analyze the various proposed mechanisms and possible intermediates.

In a second step with the same manganese-acacen' representation of the organometallic complex the two variants of the radical probe were modeled as reactants (the substituted styrenes are shown in Figure 2). With this model we aim to describe the observed distribution between epoxidation and ringopening products (Table 1). We will focus on the question whether an epoxidized olefin can be formed via a radical intermediate or if these radicals will always lead to ring opening products.

Our third model accounts for the full salen complex which was shown earlier to be important for a reliable description of the selectivity. ${ }^{[20 \mathrm{a}]}$ In this case, the steric influence of the complex is fully taken into account. For this model, important steps in the ring-opening and epoxidation of the substituted styrenes will be modeled. However, the size of this system does not allow the usage of the larger basis set in the optimization procedure. In this case the system was optimized using a $6-31+g(d)$ level of theory for $\mathrm{H}, \mathrm{C}, \mathrm{N}$ and oxygen. And on $\mathrm{Mn}$ and $\mathrm{Cl}$, a 6$311++G(2 d f, 2 p)$ basis set was used. Free energies were calculated as mentioned in the computational details.

Mechanistic investigation on the manganese-acacen'

complex with ethylene

Using ethylene as a test molecule, the free energy diagram for the oxygen transfer was calculated with the manganeseacacen' complex containing a $\mathrm{Cl}$ counter-ion. On the spin 1 and spin 2 planes the earlier proposed two step mechanism with a radical intermediate is found, on the spin 0 plane we could not find a pathway that led to oxygen transfer. The free energy diagrams are shown in Figure 5.

The barrier for the formation of the first carbon-oxygen bond s similar on the spin 1 and spin 2 plane, but since the complexes on the spin 1 plane are generally lower in energy than the ones on the spin 2 plane the first one will be preferred. The collapse of the radical into the epoxide seems to preferably take place on the spin 2 plane but the two states are energetically very close to each other. Since the Mn"'-salen complex seems to be most stable on the spin 2 plane it is likely that a spin-crossover occurs somewhere after the first transition state. A detailed energetic picture of this crossover would require more advanced calculations and is omitted here. It must be recognized that the energy differences after the formation of the first $\mathrm{C}-\mathrm{O}$ bond is small and in the following discussion both the spin 1 and spin 2 state will be shown 

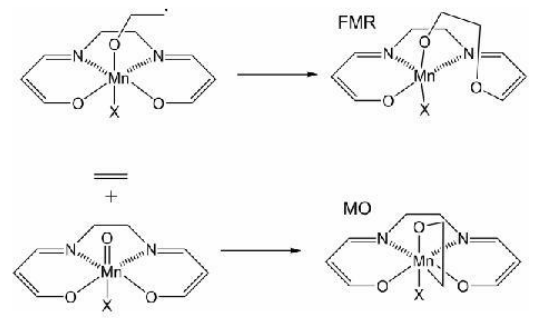

Scheme 3. Possible alternative intermediates FMR-intermediate (top), manganaoxetane intermediate (bottom)

Next to the two-step mechanism, different pathways for the oxygen transfer have been proposed in literature. The first possibility is a concerted oxygen transfer in which the epoxide is formed in a single step. However, we were unable to identify a concerted transition state. This observation is in agreement with earlier studies where the concerted step -if it could be isolatedhad a significantly higher activation barrier ${ }^{[23]}$. Other intermediates that have gained attention are the manganaoxetane structure ${ }^{[12 a]}(\mathrm{MO})$ and the five membered ring (FMR) intermediate ${ }^{[24]}$ (Scheme 3). In the first case a four membered ring is formed with the manganese, oxygen and the two carbon atoms of the olefin. In the second case a five membered ring is formed between the same atoms and one of the oxygen atoms from the complex. The different intermediates were optimized and their free energies are shown in Table 2.
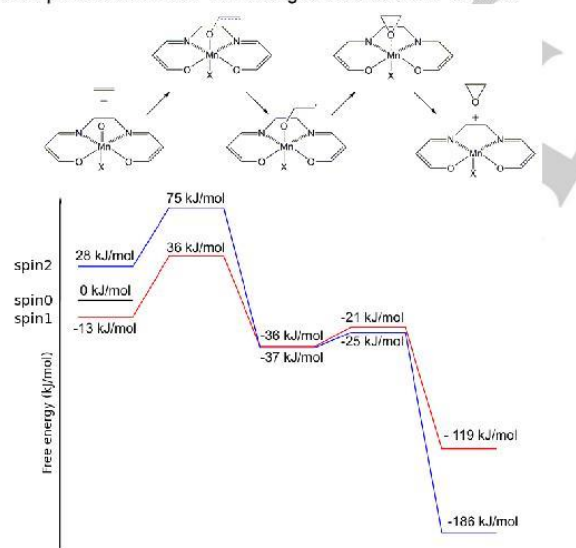

Figure 5. Free energy diagram for the oxygen transfer to ethene relative to the free energy of the reactants on the spin 0 plane $(X=C l)$.

The first possibility, the manganaoxetane structure, was only found on the spin 0 plane, it has a significantly higher free energy than other intermediates and will thus be of a limited influence. The FMR-intermediate however seems to be more stable than the radical intermediate on the spin 1 plane and could thus provide a viable alternative pathway. A search for the transition state showed there was a plausible pathway (with a barrier of $41.5 \mathrm{~kJ} / \mathrm{mol}$ ) from the radical intermediate to the FMRintermediate on the spin 1 plane, however we could not find a direct pathway to this intermediate. Moreover, a route from this intermediate to the epoxide could not be identified, making it implausible that this structure plays a significant role in the overall reaction.

Table 2. Free energies of different intermediates relative to the reactants on the spin 0 plain $(X=C l)$.

\begin{tabular}{lll}
\hline & $\begin{array}{l}\text { Spin state } \\
\text { occurance }\end{array}$ & Free energy \\
\hline & Spin 0 & $119.0 \mathrm{~kJ} / \mathrm{mol}$ \\
\hline
\end{tabular}

The study on the small manganese-acacen' complex with ethylene shows that the mechanism with the radical intermediate is the most plausible. Within this mechanism, the reaction would preferably occur on the spin 1 plane, however a spin-crossing could occur during the course of the reaction to allow formation of the most stable product. Given this observation, the radical trapping experiments of Linde at al. still needs to be explained Therefore the competition between the ring opening reaction and the epoxidation reaction starting from the radical intermediate will be studied in the next section.

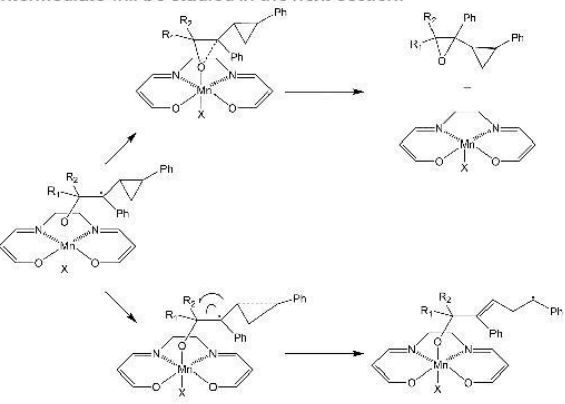

Scheme 4.Competitive pathways between epoxidation (top) and ring opening (bottom) with the radical probe starting from the radical intermediate. 

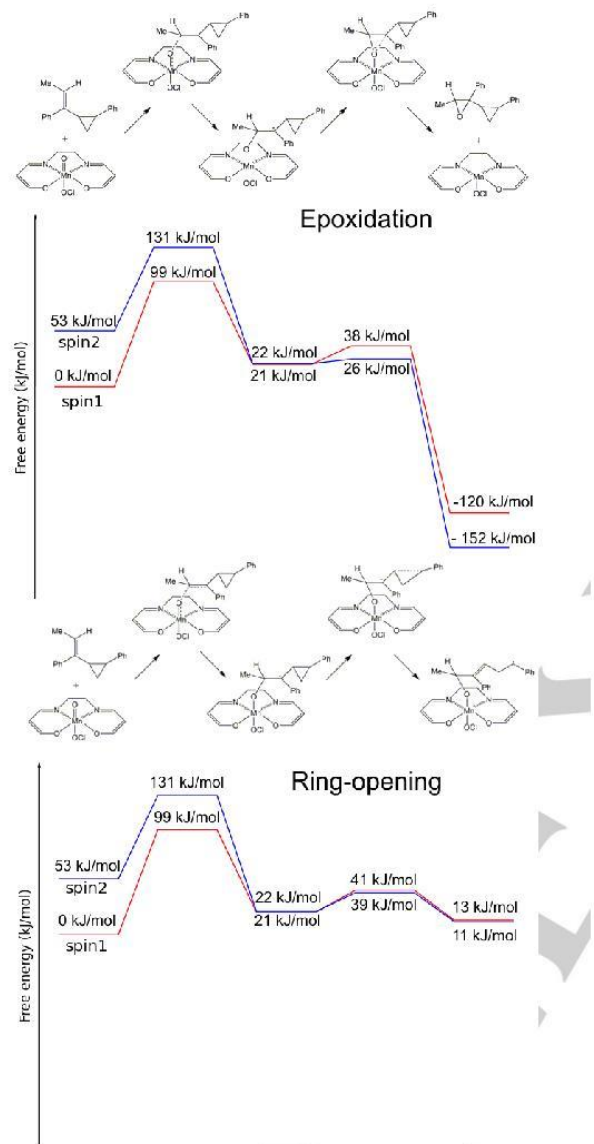

Figure 6. Epoxidation versus ring-opening pathway free energy diagram for probe (1) (nomenclature in Table 1) Free energies are relative to the reactants on spin 1.

Ring opening versus epoxidation on the manganeseacacen' complex

The experiments from Linde et al..$^{[11 b]}$ suggested that the existence of a radical intermediate should lead to a majority of ring-opening products when there is a three-membered ring adjacent to this radical (Scheme 4). In order to obtain more insight into these experimental observations, we investigated the behavior of these substrates on the Mn-acacen' model of complex. Experimental results ${ }^{[11 \mathrm{~b}]}$ showed the amount of ring-
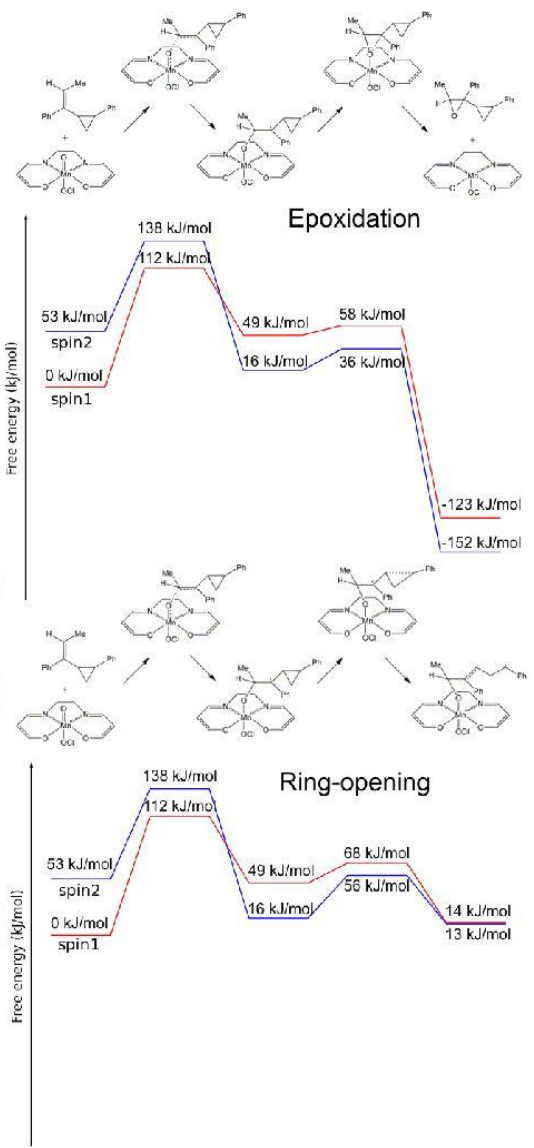

Figure 7. Epoxidation versus ring-opening pathway free energy diagram for probe (2) (nomenclature in Table 1) Free energies are relative to the reactants on spin 1.

opening products did not only vary with the exact substrate used, but also with the oxidant present (Table 1). In our models we considered two variants of the radical probe as shown in Figure 2. The dependence on the oxidant could be explained by the sixth coordination site on manganese. Jacobsen et al. ${ }^{[15]}$ showed that the counter-ion of the complex would be a hypochloride anion when the epoxidation was conducted in a sodiumhypochloride/ $\mathrm{CH}_{2} \mathrm{Cl}_{2}$ system, thus we have used this as a counter-ion in our models. 
We will study the competition between the epoxidation and ring opening reaction starting from the radical intermediate that was shown to be favored from our initial calculations. From the radical intermediate the reaction can occur via two ways: The intermediate can collapse, forming the epoxide product or the three-membered ring can open, forming a new, ring-opened intermediate. Experiments have shown that substrate (1) yields more epoxide product compared to substrate (2) (Table 1). Our theoretical results for both substrates on spin 1 and spin 2 and for both mechanisms are shown in Figure 6 and Figure 7 .

For both substrates the epoxide product is thermodynamically more favored. This is not surprising since the ring-opening reaction leads to another radical that is only an intermediate for the observed ring-opening products. From the height of the barrier we can draw some conclusions on what product is kinetically most favored. For substrate (1) the barrier

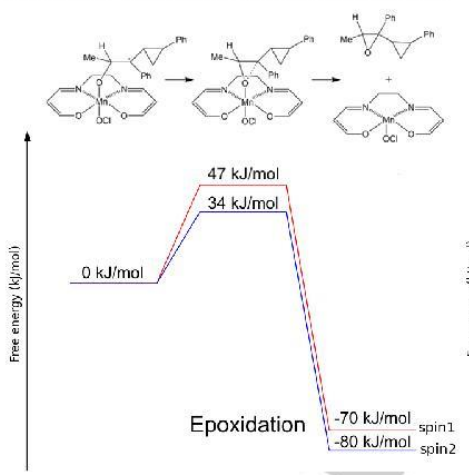

for epoxidation is about $13 \mathrm{~kJ} / \mathrm{mol}$ lower than the barrier for ringopening (Figure 6) on the most stable spin plane. This shows the epoxide formation is kinetically more favored, and it is also the experimentally observed majority product. For substrate (2) the barrier is more than $20 \mathrm{~kJ} / \mathrm{mol}$ lower in favor of the epoxide, which would mean the epoxide should also be the majority product here. However this system exhibits a fifty-fifty distribution experimentally. For the system with iodosylbenzene/benzene as oxidant/solvent combination, the results are in closer agreement with the experimental observations (Figure S1 and S2 of the supporting information) Here the epoxide product is also thermodynamically favored but with probe (2) the ring opening is kinetically favored which could correspond to the observed fifty-fifty distribution.
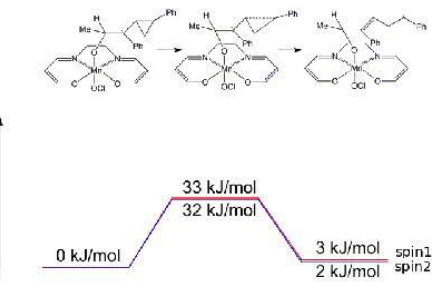

Ring-opening

Figure 8. Free energy diagrams for the competitive ring-opening and epoxidation reaction for probe (1)

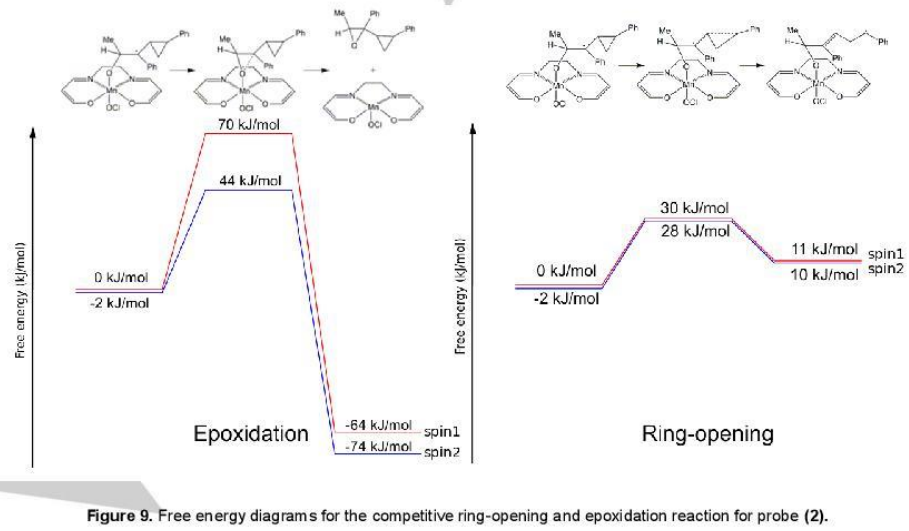


These results allow us to conclude that the radical intermediate is not necessarily 'trapped' by the ring-opening of the three-membered ring. The barrier for this opening is indeed very low, however the collapse of the radical intermediate to form the epoxide is also lowly activated, making both pathways competitive. Detailed structural parameters that explain the observations cannot easily be deduced from this relatively smal model. To fully obtain mechanistic insight, the reaction with the hypochloride anion on the sixth coordination site will be examined further using the full Jacobsen complex as a model.

\section{Ring-opening versus epoxidation on the full Jacobsen} complex

To fully account for the steric influences of the catalyst, the reaction on the full Jacobsen catalyst is modeled hereafter ${ }^{[20 a]}$ In this case the model becomes quite large and therefore the optimizations were performed using a lower basis set for part of the system, as explained in the computational details.

(1)

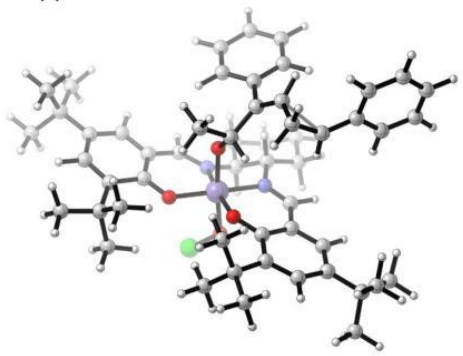

Furthermore, we will only consider the last step of the mechanism to unravel the competition between the epoxidation and ring-opening. The thermodynamic diagrams for both probes are shown in Figure 8 and Figure 9. These results give insight into the difference between the various radical probes. For probe (1) the ring-opening and epoxidation pathway have a similar barrier height $(32.3 \mathrm{~kJ} / \mathrm{mol}$ and $34.2 \mathrm{~kJ} / \mathrm{mol}$ respectively on the spin 2 plane, see Figure 8). This leads to the thermodynamically most favored epoxidation product being formed quantitatively. Probe (2), that leads experimentally to a mixture of ring-opening and epoxidized products, has a significant difference in barrier height (Figure 9). The thermodynamically favored epoxide formation has a higher free energy barrier than the ring opening, thus it is not surprising that a mixture between the thermodynamically and kinetically favored product is formed.

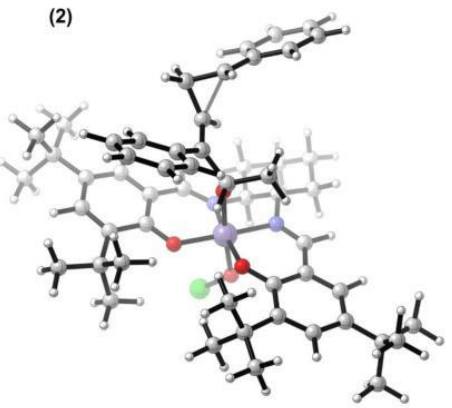

Figure 10. Geometries of the ring-opening transition states (spin 2 plane), an internal rotation in the probe occurs due to the interaction with the methyl group, but this has no influence on transition state energy.

(1)

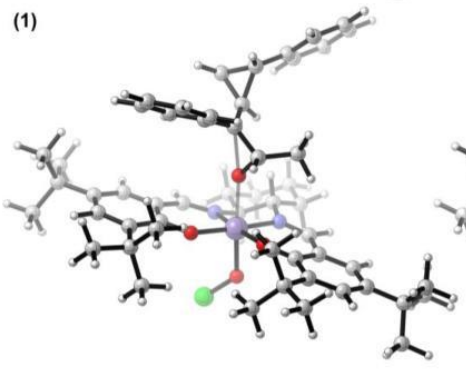

(2)

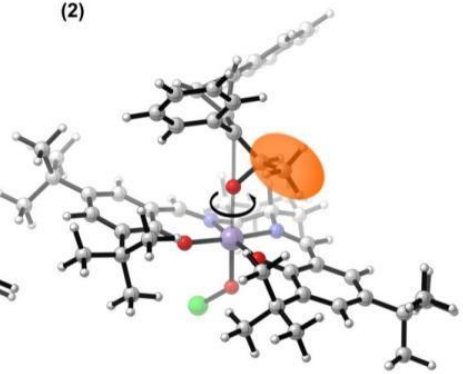

Figure 11. Geometries for the epoxidation transition states (spin 2 plane), the place of the substituents induces a steric interaction with the complex that changes the orientation between substrate and complex (rotation denoted with an arrow). This effect has an influence on the transition state. 
The difference between the behavior of the two probes can also be deduced from the geometries of the transition states for the ring-opening and the epoxidation reaction. As can be seen from the transition state for both probes, shown in Figure 10, the ring-opening reaction is not hindered by the catalytic complex, allowing the probes to assume their most favored form.

For the epoxidation reaction the nature of $R_{1}$ and $R_{2}$ substituents do seem very important due to interactions with the catalyst (Figure 11). This is according to intuition, the ringopening is not influenced by the transferred oxygen nor by the catalyst, while in the epoxidation this influence does exist, since the reaction takes place closer to the complex. With probe (1) there is no steric influence between the catalyst and the substrate during the epoxidation, this leads to a dihedral angle between the oxygen of the ligand, manganese, the transferred oxygen and the first carbon of the substrate of $7.5^{\circ}$. For probe (2) the methyl group (marked orange in Figure 11 (2)) would be hindered by the tert-butyl group of the complex, forcing the probe to twist during the transition state. This results in a dihydral angle of $133^{\circ}$ on the spin 1 plane and $60^{\circ}$ on the spin 2 plane, the twist appears to be energetically unfavorable resulting in a higher barrier. The main reason for the difference in product distribution between the probes is thus not the presence of different mechanisms but the steric influence of the complex on the transition state for the epoxidation reaction. It is logical that the epoxidation is affected more, since during the formation of the second $\mathrm{C}-\mathrm{O}$ bond the substrate occurs closer to the complex compared to the ring-opening step.

\section{Conclusions}

The nature of the intermediate during the oxidation of unfunctionalized olefins with Mn-salen complexes has triggered many experimental and theoretical studies so far. In this study we have presented a full mechanistic study using contemporary methods to give more insight into the nature of the intermediate and its repercussions on the reactions starting from it.

As the manganese salen complex is an ambitious system to describe electronically, it was necessary to first determine an electronic level of theory that was able to describe the subtle energy differences between the various spin states, whereas also being computationally feasible. Indeed, as it was our intention to study the mechanism on the full Jacobsen catalyst, an accurate yet computationally efficient method had to be selected. A computational benchmark study was performed on the Mn-acacen' complex for which recently high level DMRGSCF results became available on the stability of the various spin states. A variety of contemporary functionals were tested and hereof the OPBE functional gave an acceptable agreement with the DMRG-SCF results. In a next step we investigated all mechanistic proposals for the epoxidation reaction on the small Mn-acacen' complex with ethene as a test substrate. It was shown that the radical intermediate mechanism is the only likely candidate. At first sight, these results seem contradictory to the experimental observation using radical probes. Intuitively the opening of the three-membered ring seems more facile than the epoxidation. However for the same model system we observed that there is a competition between the epoxidation and ringopening reaction starting from the radical intermediate, which shows that the radical intermediate will in reality not necessary be trapped by the radical probe. However to fully understand the observed product distribution in terms of varying substrates and varying oxidants, it was necessary to account for the full Jacobsen catalyst and to account for all steric effects. Indeed, steric interactions between the methyl group on the radical probe and the catalyst induce a free energy difference between the transition state for epoxidation and that for ring-opening, in agreement with the experimental observations. All of these results show the most probable mechanism of oxygen transfer via a manganese-salen complex is the one featuring a radical intermediate. Starting from this intermediate both epoxidation and ring-opening can occur with the radical probes. This pathway can explain much of the experimental results, not only the distribution between cis and trans epoxides starting from cis olefins, but these results also provide an explanation for the observed product distribution between epoxidation and ringopening products when using radical probes. These conclusions are of fundamental importance for the optimizations of salen catalysts for enantioselective olefin epoxidations.

\section{Computational methodology}

As a first step a good density functional for the modelling has to be identified. Therefore a series of calculations were done with the Gaussian09 suite of programs ${ }^{[25]}$ using a variety of functionals. In the next steps all optimizations were done using a $6-311++G(2 d f, 2 p)$ basis set unless otherwise mentioned. For all results the stability of the wavefunction was checked. After optimization Grimme D3 corrections were applied to include van der Waals contributions ${ }^{[26]}$. Free energies are calculated from frequency calculations, which are done on the same level of theory as the optimizations, using the in-house TAMkin package ${ }^{[27]}$. More information on theoretical procedures to obtain kinetic data can be found in the review by Van Speybroeck et al. ${ }^{[28]}$ All transition state structures were verified by the presence of a single imaginary frequency.

\section{Acknowledgements}

The research was financed by UGent GOA grant 01G00710, the European Research Council for funding through the European Community's Seventh Framework Programme (FP7(2007-2013) ERC grant agreement no.1 240483) and the Research Board of Ghent University (BOF). S.W. acknowledges Research Foundation Flanders for a predoctoral and the Belgian-American educational foundation for a postdoctoral fellowship. Computational resources and services were provided by Ghent University (Stevin Supercomputer Infrastructure). 
Keywords: Ab initio calculations - Oxidation - Quantum chemistry $\cdot$ Manganese $\cdot$ Homogeneous catalysis

[1] a) W. Zhang, J. L. Loebach, S. R. Wilson, E. N. Jacobsen, J. Am Chem. Soc. 1990, 112, 2801-2803; b) R. Irie, K. Noda, Y. Ito, N. Matsum oto, T. Katsuki, Tetrahedron Lett. 1990, 31, 7345-7348.

[2] T. Katsuki, Coord. Chem. Rev. 1995, 140, 189-214.

[3] a) J. F. Larrow, E. N. Jacobsen, Y, Gao, Y. P. Hong, X. Y. Nie, C. M. Zepp, J. Org. Chem. 1994, 59, 1939-1942; b) M. Palucki, P. J. Pospisil W. Zhang, E. N. Jacobsen, J. Am. Chem. Soc. 1994, 116, 9333-9334 c) N. H. Lee, E. N. Jacobsen, Tetrahedron Lett. 1991, 32, 6533-6536.

[4] a) A. P. Gao, M. Wang J. C. Shi, D. P. Wang, W. Tian, L. C. Sun, Appl Organomet. Chem. 2006, 20,830-834; b) H. Egami, T. Katsuki, J. Am. Chem. Soc. 2007, 129, 8940-8941; c) A. Y. Choi, G. S. Hwang, B. K. Senapati, D. H. Ryu, Bull. Korean Chem. Soc. 2008, 29, 1879-1880.

[5] W. Zhang. E. N. Jacobsen, J. Org. Chem. 1991, 56, 2296-2298.

[6] a) G. J. Kim, S. J. Kim, H. S. Lee, D. W. Park, J. W. Yun, Google Patents, 2001; b) Y. Ishikawa, H. Eguchi, Google Patents, 2013.

[7] M. Palucki, G. J. McCormick, E. N. Jacobsen, Tetrahedron Lett. 1995 36, 5457-5460

[8] E. N. Jacobsen, W. Zhang, A. R. Muci, J. R. Ecker, L. Deng, J. Am Chem. Soc. 1991, 113, 7063-7064.

[9] D. A. Plattner, D. Feichtinger, J. El-Bahraoui, O. Wiest, Int. J. Mass Spectrom. 2000, 195, 351-362

[10] H. Fu, G. C. Look, W. Zhang, E. N. Jacobsen, C. H. Wong, J. Org Chem. 1991, 56, 6497-6500

[11] a) C. Linde, N. Koliai, P. O. Norrby, B. Akermark, Chem.-Eur. J. 2002, 8 , 2568-2573; b) C. Linde, M. Arnold, P. O. Norrby, B. Akermark, Angew. Chem.Int. Edit. Engl. 1997, 36, 1723-1725.

[12] a) C. Linde, B. Akermark, P. O. Norrby, M. Svensson, J. Am. Chem. Soc. 1999, 121, 5083-5084; b) T. Strassner, K. N. Houk, Org. Lett. $1999,1,419-421$.

[13] Y. G. Abashkin, J. R. Collins, S. K. Burt, Inorg. Chem. 2001, 40, 4040 4048

[14] a) L. Cavallo, H. Jacobsen, Eur. J. Inorg. Chem. 2003, 892-902; b) L. Cavallo, H. Jacobsen, J. Phys. Chem. A 2003, 107, 5466-5471; c) L. Cavallo, H. Jacobsen, J. Org. Chem. 2003, 68, 6202-6207

[15] L. Cavallo, H. Jacobsen, Inorg. Chem. 2004, 43, 2175-2182.

[16] I. V. Khavrutskii, D. G. Musaev, K. Morokuma, Proceedings of the National Academy of Sciences of the United States of America 2004 101, 5743-5748.

[17] a) J. S. Sears, C. D. Sherrill, J. Chem. Phys. 2006, 124, 144314; b) J. Ivanic, J. R. Collins, S. K. Burt, J. Phys. Chem. A 2004, 108, 23142323; c) D. Ma, G. Li Manni, L. Gagliardi, The Journal of Chemical Physics 2011, 135,

[18] S. Wouters, W. Poelmans, P. W. Ayers, D. Van Neck, Computer Physics Communications 2014, 185, 1501-1514.

[19] S. Wouters, T. Bogaerts, P. Van der Voort, V. Van Speybroeck, D. Van Neck, J. Chem. Phys. 2014, 140, 241103

[20] a) T. Bogaerts, A. Van Yperen-De Deyne, Y.-Y. Liu, F. Lynen, V. Van Speybroeck, P. Van Der Voort, Chem. Commun. 2013, 49, 8021-8023; b) T. Bogaerts, S. Wouters, P. Van Der Voort, V. van Speybroeck, J. Mol. Catal. A: Chem 2015, in Press.

[21] a) N. C. Handy, A. J. Cohen, Mol. Phys. 2001, 99, 403-412; b) W.-M. Hoe, A. J. Cohen, N. C. Handy, Chemical Physics Letters 2001, 341 319-328.

[22] a) M. Swart, Inorg. Chim. Acta 2007, 360, 179-189; b) M. Swart, A. R. Groenhof, A. W. Ehlers, K. Lammertsma, J. Phys. Chem. A 2004, 108 $5479-5483$

[23] a) L. Cavallo, H. Jacobsen, Angewandte Chemie International Edition 2000, 39, 589-592; b) H. Jacobsen, L. Cavallo, Phys. Chem. Chem. Phys. 2004, 6, 3747-3753.

[24] Y. G. Abashkin, S. K. Burt, Org. Lett. 2004, 6, 59-62
[25] G. W. T. M. J. Frisch, H. B. Schlegel, G. E Scuseria, M. A. Robb J. R. Cheeseman, G. Scalmani, V. Barone, B. Mennucci, G. A. Petersson, H. Nakatsuji, M. Caricato, X. Li, H. P. Hratchian, A. F. Izmaylov, et al. Revision D. 01, Gaussian, Inc., Wallingford CT ed. 2009.

[26] a) S. Grimme, J. Antony, S. Ehrlich, H. Krieg, J. Chem. Phys. 2010 132; b) S. Grimme, J. Comput. Chem. 2004, 25, 1463-1473.

27] A. Ghysels, T. Verstraelen, K. Hemelsoet, M. Waroquier, V. Van Speybroeck, J. Chem Inf. Model. 2010, 50, 1736-1750.

[28] V. van Speybroeck, R. Gani, R. J. Meier, Chem. Soc. Rev. 2010, 39 $1764-1779$ 


\section{Supporting information}

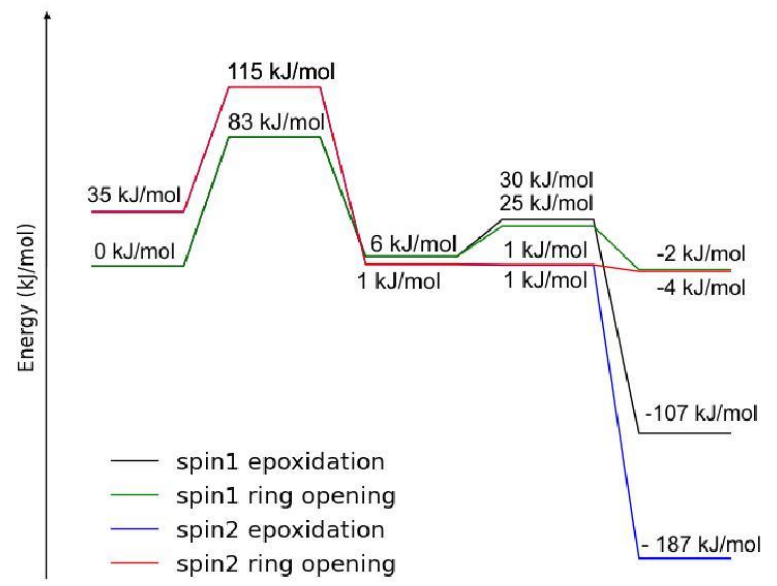

Figure S1. Epoxidation versus ring-opening pathway free energy diagram for probe (1) in the iodosylbenzene/benzene, free energies are relative to the reactants on spin 1.

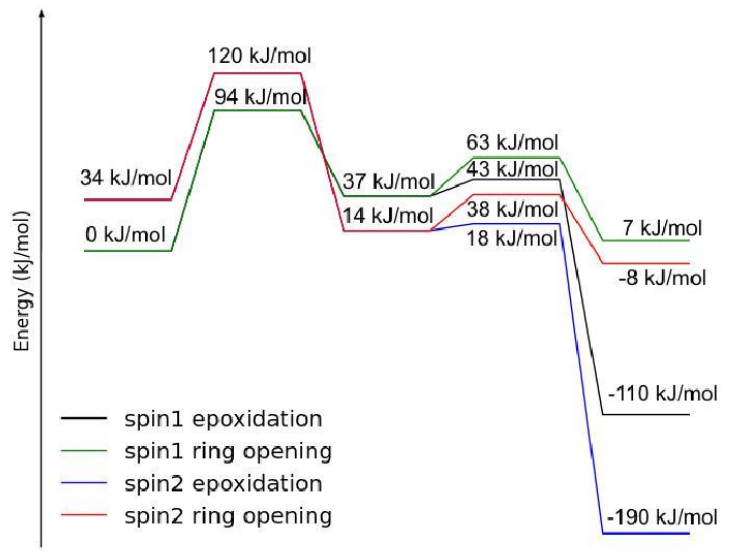

Figure S2. Epoxidation versus ring-opening pathway free energy diagram for probe (2) in the iodosylbenzene/benzene, free energies are relative to the reactants on spin 1. 
PAPer III: The EnANTioselectivity OF THE MANGaneseSALEN COMPLEX IN THE EPOXIDATION OF UNFUNCTIONALIZED OLEFINS AND THE INFLUENCE OF GRAFTING
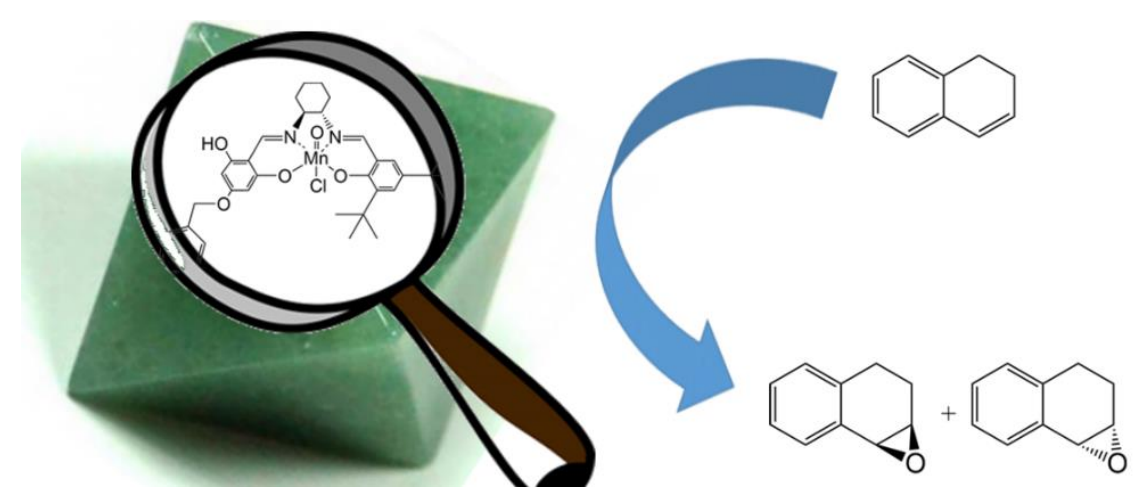

T. Bogaerts, S. Wouters, P. Van der Voort, V. Van Speybroeck, Catalysis A: Chemical, in press, 2015

T. Bogaerts performed the research and prepared the manuscript. 